\title{
BMJ Open Effect of interdisciplinary care on weight loss: a randomised controlled trial
}

\author{
Linda C Tapsell, ${ }^{1,10}$ Maureen Lonergan, ${ }^{2,10}$ Marijka J Batterham, ${ }^{3}$ \\ Elizabeth P Neale, ${ }^{1,10}$ Allison Martin, ${ }^{1,10}$ Rebecca Thorne, ${ }^{1,10}$ Frank Deane, ${ }^{4,10}$ \\ Gregory Peoples ${ }^{1,10}$
}

To cite: Tapsell LC, Lonergan M, Batterham MJ, et al. Effect of interdisciplinary care on weight loss: a randomised controlled trial. BMJ Open 2017;7:e014533. doi:10.1136/ bmjopen-2016-014533

- Prepublication history and additional material are available online. To view these files please visit the journal online (http://dx. doi.org/10.1136/bmjopen-2016014533)

Received 30 September 2016 Revised 23 February 2017 Accepted 19 May 2017

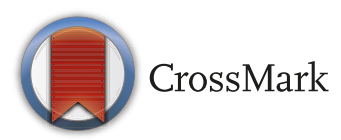

${ }^{1}$ School of Medicine, University of Wollongong, Wollongong, Australia

${ }^{2}$ Department of Renal Medicine, Illawarra Shoalhaven Local Health District, Wollongong Hospital, Wollongong, Australia ${ }^{3}$ School of Mathematics and Applied Statistics, University of Wollongong, Wollongong, Australia

${ }^{4}$ School of Psychology, Illawarra Health and Medical Research Institute, New South Wales, Australia

${ }^{10}$ |llawarra Health andMedical Research Institute, University of Wollongong, Wollongong, Australia

Correspondence to Dr Linda C Tapsell; Itapsell@ uow.edu.au

\section{ABSTRACT}

Objective To determine the effectiveness of a novel interdisciplinary treatment compared with usual care on weight loss in overweight and obese adult volunteers. Design Single blinded controlled trial. Participants randomly assigned to usual care $(C$, general guidelinebased diet and exercise advice), intervention (I, interdisciplinary protocol) or intervention + a healthy food supplement (30 g walnuts/day) (IW).

Setting Community based study, Illawarra region, south of Sydney, Australia.

Participants Generally well volunteer adult residents, 25-54 years, body mass index (BMI) $25-40 \mathrm{~kg} / \mathrm{m}^{2}$ were eligible. At baseline 439 were assessed, 377 were randomised, 298 completed the 3-month intensive phase and 178 completed the 12-month follow-up.

Interventions Treatment was provided at clinic visits intensively (0 months, 1 month, 2 months, 3 months) then quarterly to 12 months. Support phone calls were quarterly. All participants underwent blinded assessments for diet, exercise and psychological status.

Primary and secondary measures The primary outcome was difference in weight loss between baseline and 12 months (clinically relevant target $5 \%$ loss). Secondary outcomes were changes in blood pressure, fasting blood glucose and lipids, and changes in diet, exercise and psychological parameters.

Results At 12 months, differences in weight loss were identified $(p<0.001)$. The I group lost more than controls at 3 months $(91.11(92.23,90.00), p<0.05)$ and the IW more than controls at 3 months $(91.25(92.35,90.15)$, $\mathrm{p}<0.05)$ and 6 months $(92.20(93.90,90.49), \mathrm{p}<0.01)$. The proportion achieving $5 \%$ weight loss was significantly different at 3 months, 6 months and 9 months ( $p=0.04, p=0.03, p=0.03$ ), due to fewer controls on target at 3 months, 6 months and 9 months and more IW participants at 6 months. Reductions in secondary outcomes (systolic blood pressure, blood glucose/lipid parameters and lifestyle measures) followed the pattern of weight loss.

Conclusions An interdisciplinary intervention produced greater and more clinically significant and sustained weight loss compared with usual care. The intensive phase was sufficient to reach clinically relevant targets, but longterm management plans may be required.

Trial registration number ANZCTRN 12614000581662; Post-results.

\section{Strengths and limitations of this study}

- The study was closely aligned to practice and protocols tested could be readily translated into primary care services.

- Although this was a single-centre study, substantial controls were applied to provide quality evidence of effects.

- The study demonstrated the breadth of behavioural influences integral to achieving weight loss and clinical outcomes.

- Rigorous statistical analyses were applied to the evaluation of primary outcomes, including a sensitivity analysis to confirm effects.

- As practice-oriented research, retention strategies were not applied, with higher than anticipated loss to follow-up following the intensive phase.

\section{INTRODUCTION}

The prevention and management of chronic non-communicable disease (CNCD) is a challenge for health services. ${ }^{1}$ Given the links to disease pathology, identifying overweight as a problem is an important first step. ${ }^{2}$ Primary care is an ideal setting for the clinical management of obesity, yet relevant studies are scarce, ${ }^{3}$ and measuring or recording weight in this setting appears suboptimal. ${ }^{4}$ In addition, weight management may require a more shared sense of decision making, ${ }^{5}$ and a broader approach, including the expertise of relevant allied health professionals. ${ }^{6}$ For example, dietitians may provide expertise on nutritional factors other than dietary energy that influence weight loss and chronic disease risk factors, ${ }^{7}$ such as dietary patterns, ${ }^{8}$ significant foods, ${ }^{9}$ and nutrients such as fibre,${ }^{10}$ fatty acids $^{11}$ and sodium. ${ }^{12}$

Health behaviours that can significantly lower disease risk are central to the management of chronic disease.$^{13}$ There is convincing evidence that focusing on diet, physical activity and behaviour will have the best effects on overweight. ${ }^{14}$ Obese individuals who lose 
just $5 \%$ of their body weight (the target for American College of Cardiology/American Heart Association clinical guidelines ${ }^{2}$ ) have significant improvements in risk factors for type 2 diabetes and cardiovascular disease, including improved insulin sensitivity and reduced fat in the liver. ${ }^{15}$ However, there are underlying metabolic problems and weight regain invariably follows. ${ }^{16}{ }^{17}$ This suggests obesity itself is a chronic condition requiring acute effective treatments repeated at intervals ${ }^{16}$ with the provision of consistent positive reinforcement to address associated complex psychological factors. ${ }^{18}$ There is little research on holistic treatments that integrate diet, exercise and psychological support ${ }^{19}$ and research is needed to test novel protocols in this area. ${ }^{20}{ }^{21}$ In a feasibility trial comparing usual care with an interdisciplinary model, we found high eligibility (83\%) and completion $(87 \%)$ rates and a preliminary effect of $-3.98 \mathrm{~kg}$ greater weight loss over 3 months $(95 \%$ CI 6.17 to $1.79, p=0.002){ }^{22}$ The next research question was whether weight loss could be achieved in a larger cohort and over a longer time period. The objective of the current trial was to determine the effectiveness of a novel interdisciplinary treatment compared with usual care on weight loss in overweight and obese adult volunteers. We hypothesised that a model of care with physician oversight that integrates the expertise of dietitians, with exercise physiologists and psychologists will be more effective than general advice provided by a practice nurse (usual care). Further, the provision of a supplement of a significant healthy food may enhance this effect and influence the overall diet.

\section{METHODS}

\section{Study oversight}

The trial is registered with the Australian and New Zealand Clinical Trial Registry (ANZCTRN12614000581662). Study oversight was provided by the senior clinical investigative team.

\section{Study participants}

Recruitment was conducted through communications and advertising in the local media. Respondents who were permanent residents of the Illawarra region, aged 25-54 years, community dwelling and with a body mass index (BMI) $25-40 \mathrm{~kg} / \mathrm{m}^{2}$ were included. Exclusion criteria were being unable to communicate in English; having severe medical conditions impairing the ability to participate in the study or thought to limit survival to 1 year, having reported illegal drug use or regular alcohol intake associated with alcoholism ( $>50 \mathrm{~g} /$ day); or other major impediments to participation.

\section{Trial design}

This was a community-specific (single-centre), randomised, assessor blinded trial, comparing outcomes between intervention and control groups at 0 months, 3 months, 6 months, 9 months, 12 months. Full details of the study protocol and baseline results are reported elsewhere. ${ }^{23}$ Briefly, all participants attended the clinic for counselling on seven occasions (0 months, 1 months, 2 months, 3 months, 6 months, 9 months, 12 months) and received quarterly support phone calls. Assessment and treatment protocols were devised by the research team including physicians, dietitians, exercise physiologists and psychologists. Measurements were undertaken separately at these time points. Body weight $(\mathrm{kg})$ was measured at each visit in an upright position (minimal clothing, no shoes) using scales with a bioelectrical impedance component for estimating body fat (\%) (Tanita TBF-662, Wedderburn, Ingleburn, New South Wales, Australia). Systolic blood pressure (SBP) and diastolic blood pressure were measured at 0 months, 3 months and 12 months using the Omron BP-203RPEIII VP-1000 device (Omron Healthcare, Kyoto, Japan). Measurements are collected at the end of 5 min resting period in supine position. Fasting blood lipids (cholesterol, low-density lipoprotein (LDL), high-density lipoprotein (HDL), triglycerides), fasting blood glucose and serum HbAlc were assessed through a registered pathology service (Southern IML Pathology) quarterly. For 24 hours urinary sodium assessments, a 24 hours urine sample was provided at 0 months, 3 months and 12 months. Dietary intake was assessed using a diet history interview $^{24}$ and physical activity using the International Physical Activity Questionnaire (IPAQ) ${ }^{25}$ and via a pedometer (Yamax Digiwalker SW200, Pedometers Australia) worn for a 4-day period every quarter. A range of psychological assessments were applied, including the Physical and Mental Health 12-Item Short-Form Health Survey (SF-12) (12 questions), ${ }^{26}$ Depression Anxiety Stress Scale (short form 21 questions), ${ }^{27}$ and Acceptance and Action Questionnaire for Weight-related problems (11 questions) (AAQW) ${ }^{28}$ at 0 months, 3 months and 12 months.

Participants were randomly assigned to usual care $(\mathrm{C}$, general advice), intervention (I, interdisciplinary advice) and intervention + food supplement (IW, I+30 g walnuts/ day). Usual care involved a nurse providing general advice based on the Australian Guide to Healthy Eating $(\mathrm{AGHE})^{29}$ and National Physical Activity Guidelines. ${ }^{30}$ Phone contact at quarterly intervals was also made by the study administrator delivering semiscripted patient-centred support of short duration. In the intervention counselling session an accredited practising dietitian negotiated changes in specific food choices based on a diet history assessment and materials that referred to the food groups outlined in AGHE (vegetables, fruits, grains, protein rich foods, dairy foods, oils). This consultation included advice to increase physical activity and reduce sedentary behaviour by identifying opportunities in leisure, occupation and household activities, with additional categorical guidance prepared by the exercise physiologist following exercise assessment. The psychologist developed a workbook for participants and trained health coaches to deliver related scripted calls of short (15 min) duration at quarterly intervals. The psychological coaching component was based on principles from Acceptance and Commitment Therapy ${ }^{31}$ and involved 
clarification of underlying values to increase motivation related to weight loss, increasing mindfulness and awareness to facilitate better health choices, and self-compassion to promote continued valued-action even in the presence of setbacks.

Randomisation was performed remotely in randomly allocated blocks of three, six or nine by an investigator unrelated to the clinic. A computer generated randomisation sequence was used (STATA V.12, StataCorp, College Station, Texas, USA). The randomisation was stratified according to sex and BMI (low BMI $\leq 30$ and high $\mathrm{BMI}>30$ ). The randomisation list was provided to the study team who added eligible participants sequentially for each of the strata. Participants were blinded to their randomised allocation and only advised they would be seen by a health practitioner.

\section{Effectiveness outcomes}

All end points compared baseline data with 12-month results. The primary outcome was body weight $(\mathrm{kg})$. Secondary outcomes were fasting blood lipids, glucose and haemoglobin A1c (HbA1c), SBP, dietary intake, measures of physical activity and psychological wellbeing.

\section{Statistical analysis}

Outcomes were analysed using mixed models. The primary outcome variable weight was analysed using a published model building procedure. ${ }^{32}$ Initially a simple model with main effects and a group by time interaction was considered. Initial data exploration suggested quadratic and cubic terms may be needed and these were added in turn and tested with likelihood ratio tests to determine improvement in model fit. Random effects for both intercept and slope were included in the weight model. A similar procedure was followed for all other variables. Significant higher order interaction terms were followed up by analysis of covariance (ANCOVA) to determine differences between groups at each time point with baseline value as a covariate. Gender was included as a covariate in the body composition models. As the dropout rate at the end of the follow-up period (12 months) was substantial, several sensitivity analyses were performed. First multiple imputation of 100 data sets was used to verify the significance of the difference between the groups at all time points. The imputation model included group, age and gender, as well as weight at each time point. A complete case analysis, last observation carried forward (LOCF) and baseline observation carried forward (BOCF) were also performed. Model building was performed using LMER in the LME4 package of R (RStudio V.0.99.489, RStudio). Multiple Imputation was performed in SAS (V.9.4 SAS Institute, Cary, North Carolina, USA) using PROC MI, PROC MIXED and MIANALYSE, for the ANCOVA. $\mathrm{F}$ tests for the 100 multiple imputations using PROC
MIXED were combined using the package MICEADDS in $\mathrm{R}$.

\section{RESULTS}

\section{Participants}

Recruitment began in May 2014 and the last participant completed in May 2016. Surveys were sent to 718 respondents, 439 of whom underwent baseline assessments. $\mathrm{n}=377$ were randomised into the $\mathrm{C}(\mathrm{n}=126)$, I $(\mathrm{n}=125)$ and IW groups $(\mathrm{n}=126)$. The intensive phase was completed by 298 participants (withdrawal rate $18 \%$ ) and the 12 months follow-up by $\mathrm{n}=178$ participants (withdrawal rate 39\%) (figure 1). Screening and baseline data are reported elsewhere. ${ }^{23}$ The sample comprised mostly obese (BMI 32 (29-35) kg/m²), non-smoking (98\%) well educated ( $85 \%$ postschool qualifications) women (74\%) of median age 45 (37-51) years. They also suffered from anxiety $(26.8 \%)$ and depression $(33.7 \%)$ and were treated for hypertension (25\%). Metabolic syndrome was identified in $34 \%$ of participants. ${ }^{33}$

Participants attended the Clinical Trials Unit of the Illawarra Health and Medical Research Institute. After randomisation 67 participants withdrew, with most (75\%) citing an inability to commit time and/or personal reasons. The next major withdrawal $(n=49)$ occurred after the 3-month intensive phase for similar reasons. Attendance gradually reduced for all groups but IW participants attended more, and were more likely to complete the phone coaching calls than the I group (at quarters $2,3,4 ; \mathrm{p}<0.05)$. Less than a quarter of participants were on medications for glucose, lipids and blood pressure. There were no differences between groups for medication use $(\mathrm{p}>0.05)$ (table 1$)$.

\section{Primary outcomes}

\section{Weight loss}

After 12 months weight reduced in all groups with a significant difference between groups $(p=0.0002)$ (tables 2 and 3 ). The primary analysis model including group, gender and time, found a quadratic time by group interaction. The effect was seen with the IW group showing initial weight loss and then a gain from 6 months, while the other groups maintained their weight loss over time (figure 2). Post hoc analysis on complete cases indicated significantly greater weight loss in I and IW compared with $\mathrm{C}$ at 3 months $(-1.2 \mathrm{~kg}, \mathrm{p}=0.045 \mathrm{I}$; $-1.3 \mathrm{~kg}, \mathrm{p}=0.025 \mathrm{IW})$ and at 6 months for IW $(-2.1 \mathrm{~kg}$; $\mathrm{p}=0.010)$. The ANCOVA compared the groups using a mixed model on the actual data and the combined estimates for 100 imputations (table 2 and supplementary materials). A sensitivity analysis confirmed the effects (table 3). An ANCOVA on complete cases for the 12-month weight change adjusted for baseline weight, gender and age showed an effect approaching significance $\mathrm{p}=0.056$ reflecting a difference between the C-IW group of $-2.2 \mathrm{~kg}$ ( $95 \% \mathrm{CI}-4.6$ to $0.1 \mathrm{~kg}, \mathrm{p}=0.068$ ) compared with differences between the C-I groups 


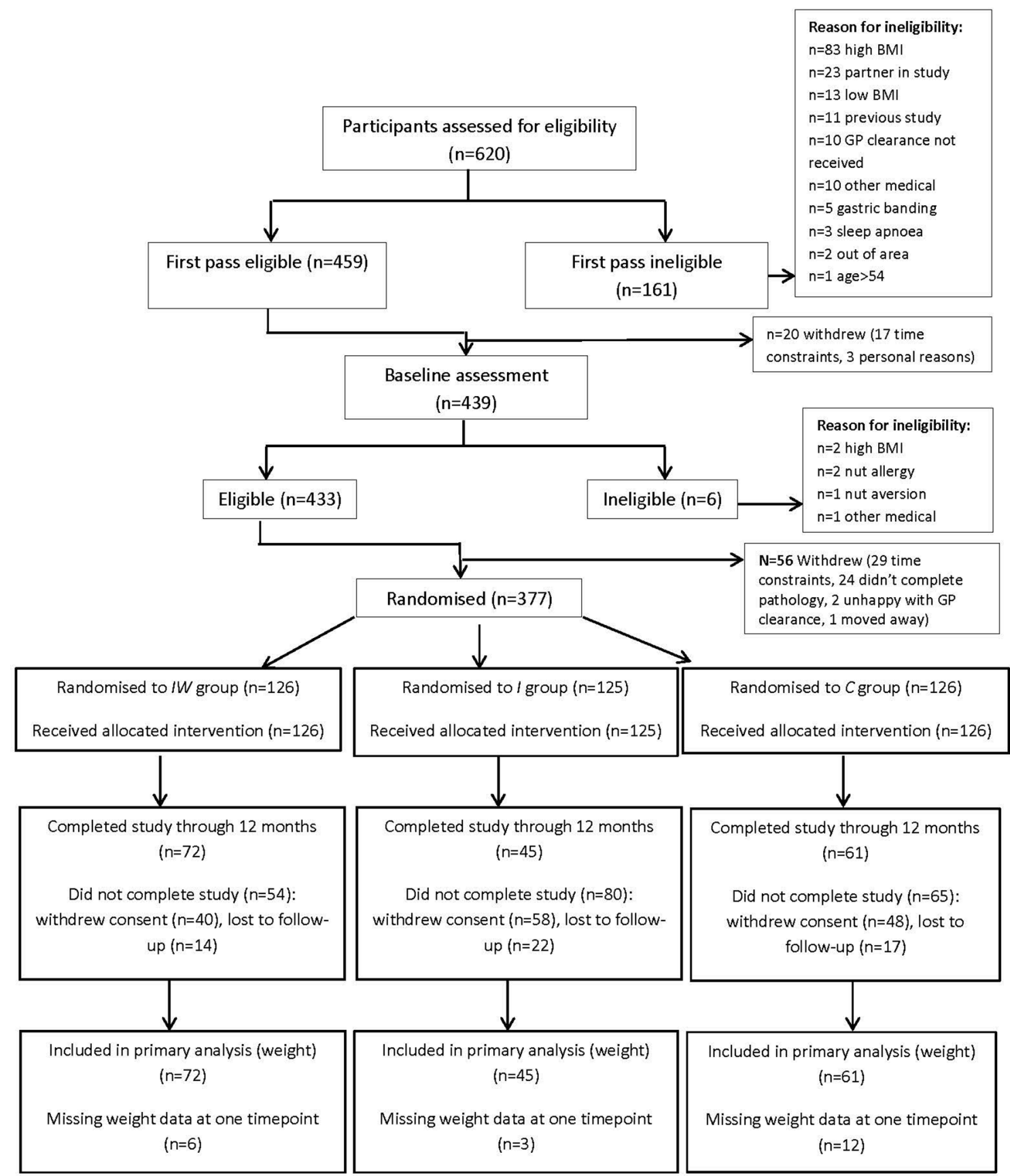

Figure 1 Participant flow in the HealthTrack randomised controlled trial.

$-1.9 \mathrm{~kg}(95 \% \mathrm{CI}-4.5$ to $0.7 \mathrm{~kg}, \mathrm{p}=0.228)$ and the $\mathrm{I}$ and IW groups $-0.3 \mathrm{~kg}(95 \% \mathrm{CI}-2.8$ to $2.2 \mathrm{~kg}, \mathrm{p}=1.00)$.

Achievement of $5 \%$ weight loss target

$\chi^{2}$ Analyses indicated significant differences in the proportion of participants achieving the clinically significant effect of $5 \%$ weight loss. At 3 months, 6 months and 9 months the proportion achieving $5 \%$ weight loss in the $\mathrm{C}$ group was lower than expected $(\mathrm{p}=0.04,0.03$, 0.03 , respectively), although there was no difference at 12 months $(\mathrm{p}=0.091)$ with $33 \%$ IW, $38 \%$ I and $20 \% \mathrm{C}$ meeting the $5 \%$ target. At 6 months the number in the IW group was higher than expected $(\mathrm{p}=0.03)$, consistent with the primary analysis. Likewise there was a group difference in change in per cent body fat (interaction effect $\mathrm{p}=0.022$ ) (table 2).

\section{Secondary outcomes}

Clinical effects

SBP decreased between baseline and 3 months but then remained unchanged (table 2). Changes in SBP also reflected patterns of sodium excretion (a marker of dietary intake) decreasing from baseline to 3 months $(p<0.001)$ and increasing to 12 months $(p=0.002)$. Likewise, fasting blood glucose was lower than baseline at 3 months $(p=0.040)$ and 6 months $(p<0.001)$, and then remained lower than 12 months $(p=0.003)$. HbAlc at 12 months was lower than the baseline value $(\mathrm{p}=0.031)$. 
Table 1 Number (\%) of participants reporting medication during the HealthTrack study

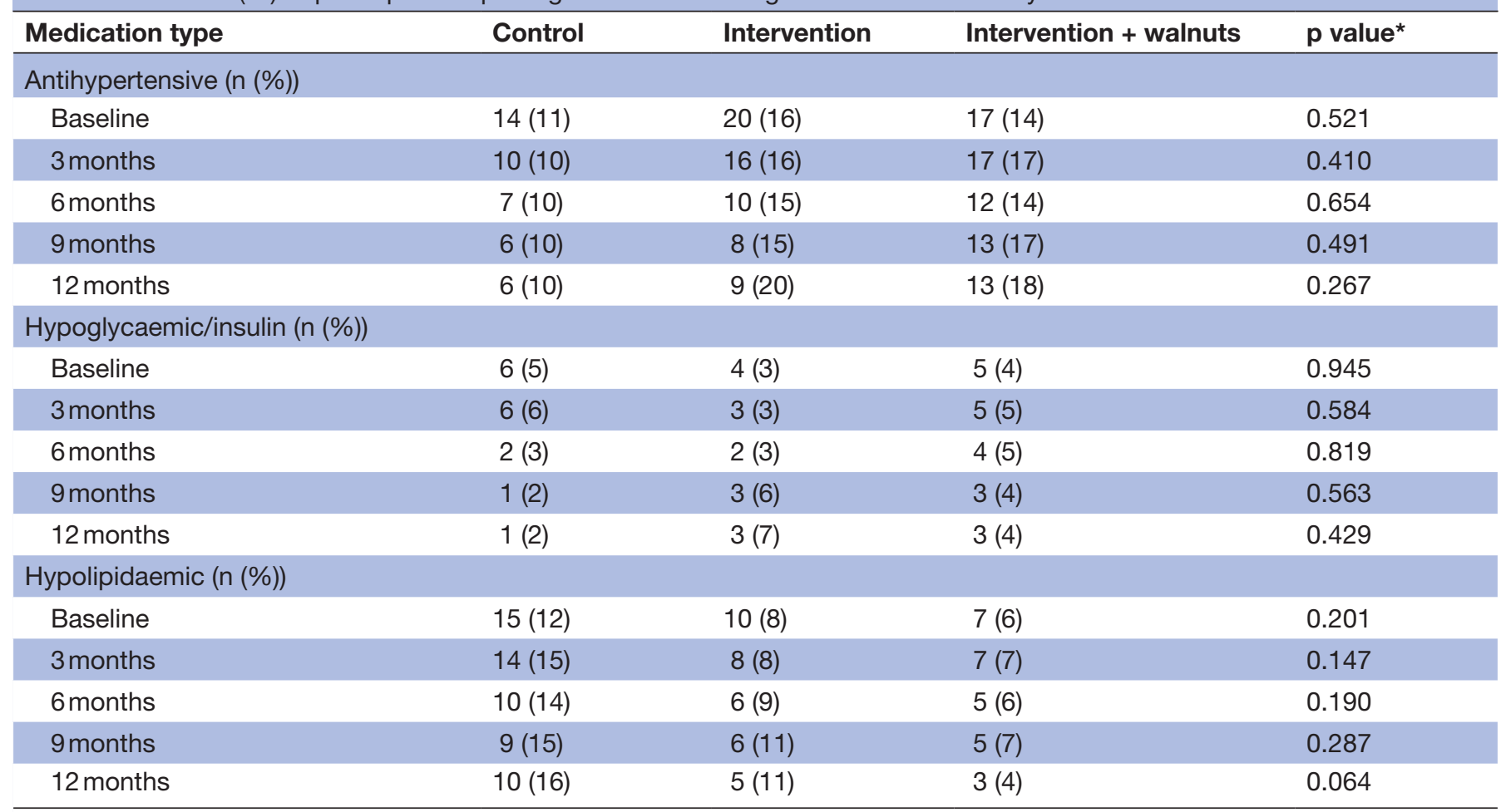

${ }^{*} \chi^{2}$ test

In keeping with this pattern of effects, total cholesterol and LDL concentrations were lowest for the sample at 3 months $(\mathrm{p}<0.001 ; \mathrm{p} \leq 0.031$, respectively $)$ and at 6 months they remained lower than baseline $(p=0.020$; $\mathrm{p}=0.034$, respectively). The cholesterol: HDL ratio decreased particularly after 6 months, while HDL- values first dropped at 3 months then returned to greater than baseline at 12 months $(\mathrm{p} \leq 0.021)$. The group effect for total cholesterol showed a lower overall mean for the IW group compared with controls $(\mathrm{p}=0.001)$ and $\mathrm{I}(\mathrm{p}=0.037)$ (table 2).

\section{Behavioural effects}

As with the pattern of weight change, reported energy and total fat intakes (as a per cent of energy) were lower than baseline at 3 months $(\mathrm{p}<0.001)$ and still at 12 months $(\mathrm{p}<0.001)$, but they increased between 3 months and 12 months $(p=0.020)$ (table 2$)$. Changes in per cent energy from protein were the opposite for dietary fat. The value was higher than baseline at 3 months and 12 months $(p<0.001)$, but lower at 12 months than 3 months $(p=0.04)$. Per cent energy from carbohydrate reduced from baseline to 12 months $(p=0.002)$, and from alcohol $(p=0.012)$ decreased from baseline to 12 months $(p=0.041)$. The only reported difference between groups was for the polyunsaturated:saturated (P:S) fatty acid ratio, where the the IW group showed a higher value over time compared with the other groups (interaction effect $\mathrm{p}<0.001)$.

The time effects for increased physical activity were stronger in self-reported MET-Mins/week (IPAQ) $(\mathrm{p}<0.001$; significantly higher than baseline at all time points, table 2) than measurements of steps / day ( $p=0.046)$ (supplementary materials). The changes in diet and physical activity were accompanied by increases in scores for positive psychological parameters (Quality of Life, QoL, table 2) and decreases for negative parameters (DASS21; AAQW, supplementary materials). The IW group scored highest for QoL (SF-12) physical summary scores throughout the study period (group effect $\mathrm{p}=0.027$ ). The QoL (SF-12) mental summary score increased after 3 months, with differences from baseline to 12 months (time effect $\mathrm{p}=0.002$ ). The DASS-21 and AAQW scores were lower at 12 months $(\mathrm{p}<0.001)$ but the significant decreases occurred at 3 months $(\mathrm{p}<0.001)$ (supplementary materials).

\section{DISCUSSION}

\section{Main findings}

Despite the same intensity of intervention and a focus on national diet and physical activity guidelines, the interdisciplinary protocol produced greater and more clinically significant effects on weight loss than usual care (figure 2). Although there were repeated attempts to retrieve participants who failed to attend visits, attrition was higher than expected, so multiple approaches including random effects mixed model analyses were applied as this would assure quality reporting required of clinical trials. ${ }^{34}$ The ANCOVA compared the groups using a mixed model on the actual data and the combined estimates for 100 imputations (table 2 and supplementary 


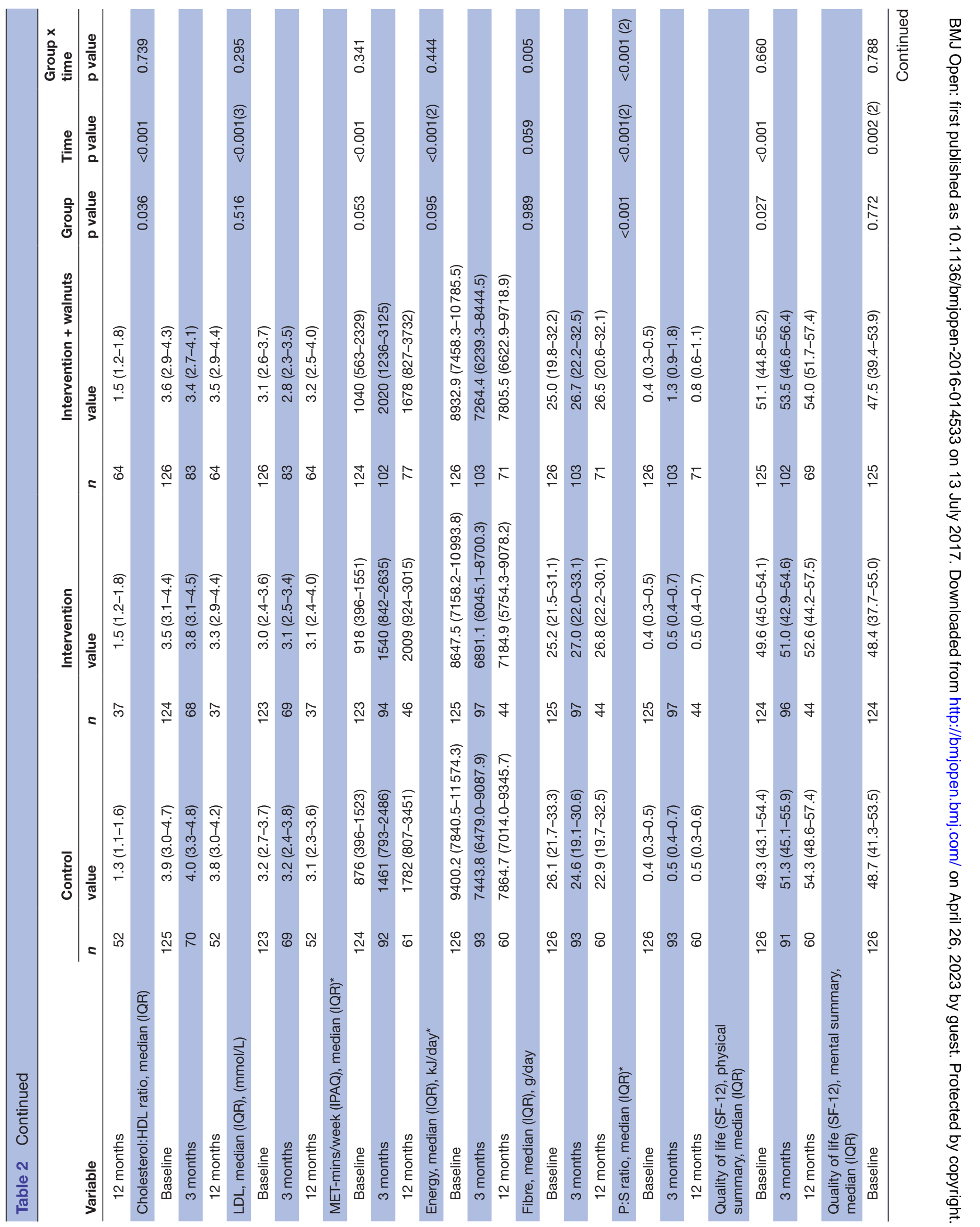



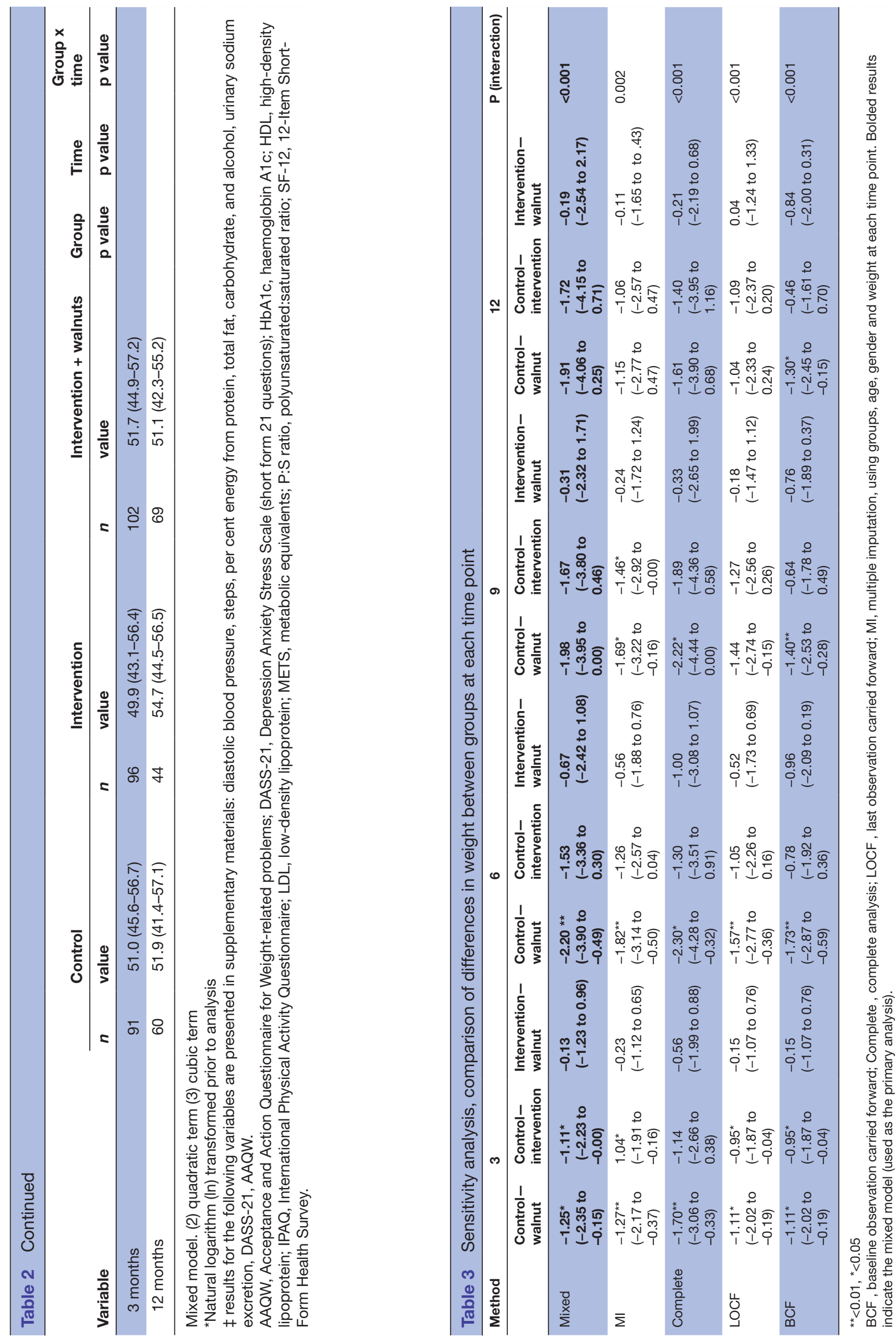

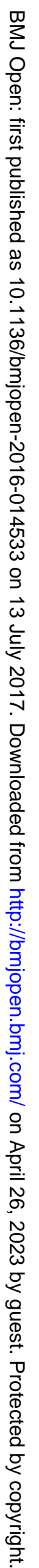



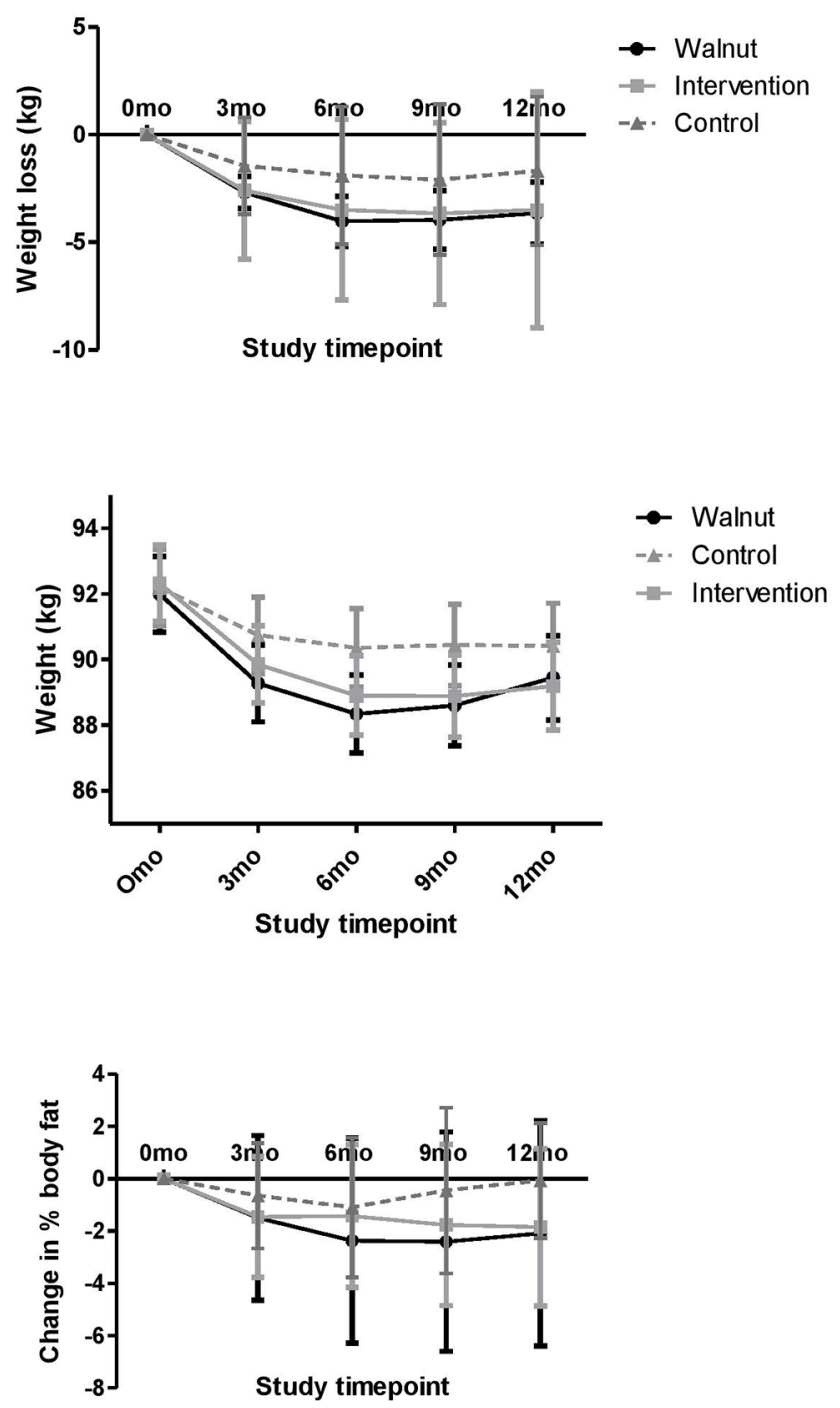

Figure 2 Difference in change in weight, weight change, and $\%$ body fat over time.

materials). A sensitivity analysis confirmed the effects (table 3) using multiple imputation (using groups, age, gender and weight at each time point), LOCF and BOCF techniques. As interaction terms were significant using all approaches, we were confident of the effects observed.

While it is not possible to separate out the components of the interdisciplinary approach, it appears more individualised advice including a focus on specific foods may have enhanced the effect. This was especially evident with the food supplemented group who continued to produce a greater weight loss at 6 months. The size of the effect and the time taken to achievement are also highly relevant to practice. The effects were similar to those reported in a systematic review of weight loss interventions which found that for combined diet and exercise interventions ranging from 12 months to 18 months, mean weight losses ranged from $0.3 \mathrm{~kg}$ to $5.9 \mathrm{~kg}$ for women and $4.2-7.3 \mathrm{~kg}$ for men. ${ }^{35}$ Without unusual retention strategies, we found that a 3-month commitment to an intensive treatment was feasible, and in that time the intervention protocol delivered a greater proportion with a $5 \%$ weight loss target. In
Western societies, it is estimated that the adult population gains $0.45 \mathrm{~kg}$ weight $/$ year, ${ }^{36}$ so our effects could be interpreted as even greater. Had we continued with monthly rather than quarterly clinic visits after 3 months we may have improved retention and study power, but that would meant greater healthcare costs. A simple sample size calculation based on the differences between groups for the completers at 12 months indicates that approximately 124 subjects per group would be required to complete the study for differences between the three groups to be statistically significant when adjusted for multiple comparisons.

\section{Secondary outcomes}

We confirmed the observation that a $5 \%$ weight loss can have an impact on disease risk factors. ${ }^{15}$ Significant reductions in SBP occurred with weight loss, as expected, but this also occurred with increased physical activity, improved mental health scores and a reduction in urinary sodium, a dietary factor known to be associated with blood pressure. ${ }^{37}$ The latter implies that the dietary changes went beyond that of energy restriction. As the national dietary guidelines were a reference point for all groups, differences in sodium intakes were not observed in this intention-to-treat analysis. Per protocol analyses may be able to detect whether greater changes occurred in the groups with the dietitian (I and IW) confirming effects seen in other primary care studies. ${ }^{38}$ Similarly the improvements in blood glucose parameters occurred with weight loss in the presence of increased physical activity and a reduced carbohydrate load for the study cohort. Further research on the types of carbohydrate-rich foods may be informative in detecting more specific differences between groups.

The changes in blood lipids were as expected with changes in weight. The lower overall mean for total cholesterol for the IW group occurred in the presence of a significantly different dietary P:S ratio. We have previously shown that integrating walnuts in an energy controlled diet can change the dietary P:S ratio with concomitant effects on lipids. ${ }^{39}$ Given that walnuts are a fat-rich food, their inclusion in the dietary modelling for the IW group would be expected to influence the overall diet profile.

\section{Implications for practice}

Practice involves an integration of evidence on many factors, and in this research we examined a number of components. We confirmed that changes in disease risk factors occurred alongside changes in body weight, physical activity, mental health scores and dietary factors known to have an impact on disease risk such as dietary sodium, fibre and fatty acid profile. ${ }^{10121640}$ In this trial the dietitian provided face-to-face counselling with participants. Being more specific about actual foods to be consumed may be more effective and providing significant healthy food (walnuts) emphasised this point. While the effects of walnuts in the diet can be found in 
the literature, ${ }^{3941}$ there may have been synergistic effects with psychological factors in our trial. The reduced psychological avoidance of weight related issues (AAQW scores) was particularly relevant and further analyses of our data may clarify the effects of health coaching when integrated into diet and physical activity advice. In addition, and based on our previous research, ${ }^{42}$ the greater initial weight loss achieved by the IW group may have influenced retention, and this may have also resulted in the higher QoL scores, but it is difficult to determine if the provision of the food supplement alone acted as the main incentive. ${ }^{43}$ The greater attendance at phone coaching sessions by the IW group, which targeted skills in mindfulness and acceptance, also may have helped deal with the stress associated with achieving health goals. ${ }^{18}$ It is difficult to tease out any singular effect as there is so much interdependence between behavioural factors, but this study has helped expose significant elements. The pattern of weight loss reflected reduced energy intake and increased physical activity (table 2), providing evidence for applying expertise in both diet and exercise. ${ }^{44}$ As sources of nutrients, the food choices drove nutritional changes underpinned by the involvement of dietitians. ${ }^{46} 47$

\section{Strengths and limitations}

The sample comprising volunteers from the community attending a single clinic was a limitation, and, although the proportion of individuals not speaking English well in the primary recruitment area was relatively low (approximately $3 \%),{ }^{48}$ excluding these individuals may have further minimised access to a high-risk group. As a case study in planning services, however, the recruitment strategy gave us an indication of who might attend for these types of treatment. The study was testing an approach applicable to primary care, so the analysis was conducted on an intention-to-treat basis rather than on compliance to treatment. In addition there was a high level of control of potential confounding variables. The design where all groups received the same intensity of intervention with dietary advice referring to foods in $\mathrm{AGHE}^{29}$ may have masked our ability to show true effects. In similar highly controlled circumstances it has been argued that for every $\mathrm{kg}$ increase in weight loss by controls, treatment effects may be reduced by about $0.3 \mathrm{~kg}^{49}$.

While weight loss was observed, the lack of betweengroup differences in reported energy intakes may reflect inaccuracies in dietary reporting and limitations in databases for estimating food energy. For example, the available energy from walnuts has been measured as $20 \%$ less than conventional estimates, ${ }^{50}$ and this may relate to other whole foods. ${ }^{51}$ The between-group differences in weight loss are plausible from the literature. ${ }^{852}$ Like other research in this area, ${ }^{85}$ this study confirms the benefit of thinking beyond energy restriction, where other dietary factors act in synergy to influence outcomes.
The trial was aligned to translation to practice, so we did not employ enhanced retention strategies, but we know that early weight loss and age $>50$ years may predict retention. ${ }^{42}$ While we observed considerable participant dropout which was predominantly due to time constraints and personal reasons, participant dropout is common in weight loss trials. ${ }^{54} \mathrm{We}$ also compared a number of missing data analysis techniques via sensitivity analyses. ${ }^{54}$ Research indicates that psychological and behavioural factors appear more aligned with attrition than other background participant characteristics, ${ }^{55}$ something we aim to study further with this data set. In the evaluation survey of the trial, participants indicated general approval of the approach and the three most listed positive features were individual attention, the health practitioner and the education provided (data not shown). Research indicates that, as part of chronic disease management, avoidance of weight gain may reduce healthcare costs in the long term. ${ }^{56}$ Four visits within the 3-month model of care could fit within the current annual Australian Medicare arrangements, ${ }^{57}$ although with considerations for eligibility, and possible co-payments. These aspects all require confirmatory research. Research is also needed on whether attending for 3 months would be sufficient to achieve this initial target, acknowledging that a 'flattening' of effects after 6 months is typical and reflects metabolic and behavioural adaptations. ${ }^{16} 17$

This study addressed a research gap providing evidence for developing effective healthcare teams in chronic disease management. ${ }^{20}{ }^{21}$ Further analyses will be able to examine motivation and commitment barriers that both participants and healthcare teams must face. It is acknowledged that addressing long-term behaviour change is difficult in primary care, ${ }^{58}$ and that a lack of motivation and incentives may hinder trials on novel lifestyle interventions. ${ }^{59}$ Our trial recruited from the community, but medical supervision and communications with primary care physicians was part of the safety management, and provided insights into translation.

\section{Conclusion}

The primary care context provides many opportunities for dealing seriously with weight management as a health issue. Excess body weight is linked to the pathology of major CNCD, and is influenced by both physiological and behavioural factors. More research with greater consolidation of interdisciplinary expertise, and establishing greater integration with medical and nursing practices will assist translation into primary care. Familiarity in standards of operation for the various professions building a full appreciation of knowledge and skills is required. Promoting opportunities to collaborate and providing guidelines ${ }^{60}$ are a start to developing long-term plans. 
Acknowledgements The authors thank the following individuals for their contributions: Professor Joseph Ciarrochi PhD, Australian Catholic University (development of health coaching intervention); Professor David Steel PhD, University of Wollongong (survey methodology and study design); Dr Joanna Russell PhD, University of Wollongong (data collection and analysis); Dr Yasmine Probst PhD, University of Wollongong (dietary modelling and dietary quality audit design); Dr Anne McMahon PhD, University of Wollongong (delivery of health coaching intervention); Sarah Leger BSc (Hons), University of Wollongong (delivery of health coaching intervention); Kate Battocchio APD AEP, University of Wollongong/ Illawarra Health and Medical Research Institute (intervention delivery and set up of procedures relating to the exercise testing and prescription); Rhoda Ndanuko APD, University of Wollongong (intervention delivery); Cinthya Wibisono APD, University of Wollongong (intervention delivery); Stacey Fuller APD, University of Wollongong (intervention delivery); Nicole Linolli APD, University of Wollongong (intervention delivery); Amy Vero APD, University of Wollongong/lllawarra Health and Medical Research Institute (intervention delivery); Patricia Bate-Charlton GCerthLM, University of Wollongong/lllawarra Health and Medical Research Institute (usual care delivery), Fiona Roberts, RN BA Grad DipEd, University of Wollongong/llawarra Health and Medical Research Institute (usual care delivery), Fiona Love BNursing, University of Wollongong/lllawarra Health and Medical Research Institute (usual care delivery) Lara Tramacco MANP, University of Wollongong/lllawarra Health and Medical Research Institute (usual care delivery); Sarah Louise Gallagher BNurse, University of Wollongong/lllawarra Health and Medical Research Institute (usual care delivery); Krystal Sattler MPsyc(Clin), University of Wollongong (data analysis); Karumathil Murali FRACP, Wollongong Hospital (medical oversight); Lauren Houston BNutSci, University of Wollongong (data quality auditing).

Contributors Study concept and design: LCT, ML, MJB, FD, GP. Acquisition, analysis, or interpretation of data: LCT, ML, MJB, FD, GP, AM, EPN, RT. Drafting of the manuscript: LCT, MJB. Critical revision of the manuscript for important intellectual content: All authors. Statistical analysis: MJB. Obtained funding: LCT. Administrative, technical or material support: LCT, ML, MJB, FD, GP, AM, EPN, RT. Study supervision: LCT, ML, AM.

Funding Illawarra Health and Medical Research Institute and the California Walnut Commission (supplementary).

\section{Competing interests None declared}

Patient consent This article does not contain personal medical information about an identifiable living individual.

Ethics approval The study was approved by the University of Wollongong/lllawarra ShoalhavenLocal Health District Human Research Ethics Committee (Health and Medical) (HE13/189) and conducted in compliance with the Principles of the Declaration of Helsinki.

Provenance and peer review Not commissioned; externally peer reviewed.

Data sharing statement Those interested in the data should contact the corresponding author

Open Access This is an Open Access article distributed in accordance with the Creative Commons Attribution Non Commercial (CC BY-NC 4.0) license, which permits others to distribute, remix, adapt, build upon this work non-commercially, and license their derivative works on different terms, provided the original work is properly cited and the use is non-commercial. See: http://creativecommons.org/ licenses/by-nc/4.0/

(C) Article author(s) (or their employer(s) unless otherwise stated in the text of the article) 2017. All rights reserved. No commercial use is permitted unless otherwise expressly granted.

\section{REFERENCES}

1. Daar AS, Singer PA, Persad DL, et al. Grand challenges in chronic non-communicable diseases. Nature 2007:450:494-6.

2. Jensen MD, Ryan DH, Apovian CM, et al. 2013 AHA/ACC/TOS guideline for the management of overweight and obesity in adults: a report of the American College of Cardiology/American Heart Association Task Force on Practice guidelines and the Obesity Society. J Am Coll Cardiol 20142014;63(25 Pt B):2985-3023.

3. McHale CT, Laidlaw AH, Cecil JE. Direct observation of weightrelated communication in primary care: a systematic review. Fam Pract 2016;33:327-45

4. Ghosh A. Depressed, anxious and breathless missing out: Weight screening in general practice in a regional catchment of New South Wales. Aust J Rural Health 2016;24:246-52.
5. Beaulac J, Sandre D. Critical review of bariatric surgery, medically supervised diets, and behavioural interventions for weight management in adults. Perspect Public Health 2017;137:162-72.

6. Huseinovic E, Bertz F, Leu Agelii M, et al. Effectiveness of a weight loss intervention in postpartum women: results from a randomized controlled trial in primary health care. Am J Clin Nutr 2016;104:362-70.

7. Tapsell LC, Neale EP, Satija A, et al. Foods, Nutrients, and Dietary Patterns: Interconnections and Implications for Dietary Guidelines. Adv Nutr 2016;7:445-54.

8. Estruch R, Martínez-González MA, Corella D, et al. Effect of a highfat Mediterranean diet on bodyweight and waist circumference: a prespecified secondary outcomes analysis of the PREDIMED randomised controlled trial. Lancet Diabetes Endocrinol 2016;4:666-76

9. Mayhew AJ, de Souza RJ, Meyre D, et al. A systematic review and meta-analysis of nut consumption and incident risk of CVD and allcause mortality. Br J Nutr 2016;115:212-25.

10. Threapleton DE, Greenwood DC, Evans CEL, et al. Dietary fibre intake and risk of cardiovascular disease: systematic review and meta-analysis. BMJ 2013;347:f6879.

11. Jakobsen MU, O'Reilly EJ, Heitmann BL, et al. Major types of dietary fat and risk of coronary heart disease: a pooled analysis of 11 cohort studies. Am J Clin Nutr 2009;89:1425-32.

12. Aburto NJ, Ziolkovska A, Hooper L, et al. Effect of lower sodium intake on health: systematic review and meta-analyses. BMJ 2013;346:346.

13. Orenstein L, Chetrit A, Dankner R. Healthy Lifestyle Pattern is protective against $30-\mathrm{Yr}$ Cancer incidence in men and women: a Cohort Study. Nutr Cancer 2016;68:410-9.

14. National Health and Medical Research Council. Clinical practice guidelines for the management of overweight and obesity in adults, adolescents and children in Australia. Melbourne: National Health and Medical Research Council, 2013.

15. Magkos F, Fraterrigo G, Yoshino J, et al. Effects of Moderate and Subsequent Progressive Weight Loss on Metabolic Function and Adipose Tissue Biology in Humans with Obesity. Cell Metab 2016;23:591-601.

16. Hall KD, Jordan PN. Modeling weight-loss maintenance to help prevent body weight regain. Am J Clin Nutr 2008;88:1495-503.

17. Fothergill E, Guo J, Howard L, et al. Persistent metabolic adaptation 6 years after "The Biggest Loser" competition. Obesity 2016;24:1612-9.

18. Blackledge J, Ciarrochi J, Deane F, eds. Acceptance and Commitment therapy: contemporary Theory Research and Practice. Bowen Hills: Australian Academic Press, 2009.

19. Tapsell LC, Neale EP. The Effect of Interdisciplinary Interventions on Risk Factors for Lifestyle Disease: A Literature Review. Health Educ Behav 2016;43:271-85.

20. Brug J, Oenema A, Ferreira I. Theory, evidence and Intervention Mapping to improve behavior nutrition and physical activity interventions. Int J Behav Nutr Phys Act 2005;2:2.

21. Glasgow RE, Emmons KM. How can we increase translation of research into practice? types of evidence needed. Annu Rev Public Health 2007;28:413-33.

22. Tapsell LC, Thorne R, Batterham M, et al. Feasibility of a communitybased interdisciplinary lifestyle intervention trial on weight loss (the HealthTrack study). Nutrition \& Dietetics 2015.

23. Tapsell LC, Lonergan M, Martin A, et al. Interdisciplinary lifestyle intervention for weight management in a community population (HealthTrack study): Study design and baseline sample characteristics. Contemp Clin Trials 2015;45.

24. Martin GS, Tapsell LC, Denmeade S, et al. Relative validity of a diet history interview in an intervention trial manipulating dietary fat in the management of type II diabetes mellitus. Prev Med 2003;36:420-8.

25. Craig CL, Marshall AL, Sjöström M, et al. International physical activity questionnaire: 12 -country reliability and validity. Med Sci Sports Exerc 2003;35:1381-95.

26. Ware J, Kosinski M, Keller SD. A 12-Item Short-Form Health survey: construction of scales and preliminary tests of reliability and validity. Med Care 1996;34:220-33.

27. Lovibond SH, Lovibond PF. Manual for the depression anxiety stress scales. 2nd edition. Sydney: edPsychology Foundation, 1995.

28. Lillis J, Hayes SC. Measuring avoidance and inflexibility in weight related problems. International Journal of Behavioral Consultation and Therapy 2008;4:348-54.

29. National Health and Medical Research Council. Australian Dietary guidelines. Canberra: National Health and Medical Research Council 2013.

30. Commonwealth Department of Health and Aged Care. Physical Activity Guidelines for Australians - Scientific Background Report. 
A report by the University of Western Australia and The Centre for Health Promotion and Research. Secondary Physical Activity Guidelines for Australians - Scientific Background Report. A report by the University of Western Australia and The Centre for Health Promotion and Research. http://www.health.gov.au/internet/main/ publishing.nsf/Content/phd-physical-guidelines-scientific-cnt.htm

31. Lillis J, Kendra KE. Acceptance and Commitment therapy for weight control: model, evidence, and future directions. J Contextual Behav Sci 2014;3:1-7.

32. Chen D, Peace K. Clinical Trial Data analysis using R. Boca Raton, FL: CRC Press, Taylor \& Francis Group, 2011.

33. Alberti KG, Eckel RH, Grundy SM, et al. Harmonizing the metabolic syndrome: a joint interim statement of the International Diabetes Federation Task Force on Epidemiology and Prevention; National Heart, lung, and Blood Institute; American Heart Association; World Heart Federation; International Atherosclerosis Society and International Association for the study of obesity. Circulation 2009;120:1640-5.

34. Nich C, Carroll KM. Intention-to-treat meets missing data: implications of alternate strategies for analyzing clinical trials data. Drug Alcohol Depend 2002;68:121-30.

35. Williams RL, Wood LG, Collins CE, et al. Effectiveness of weight loss interventions--is there a difference between men and women: a systematic review. Obes Rev 2015;16:171-86.

36. Mozaffarian D, Hao T, Rimm EB, et al. Changes in diet and lifestyle and long-term weight gain in women and men. $N$ Engl $\mathrm{J} \mathrm{Med}$ 2011;364:2392-404.

37. He FJ, Li J, Macgregor GA. Effect of longer term modest salt reduction on blood pressure: cochrane systematic review and metaanalysis of randomised trials. BMJ 2013;346:f1325.

38. Korhonen M, Kastarinen M, Uusitupa M, et al. The effect of intensified diet counseling on the diet of hypertensive subjects in primary health care: a 2-year open randomized controlled trial of lifestyle intervention against hypertension in eastern Finland. Prev Med 2003;36:8-16.

39 Tapsell LC, Gillen LJ, Patch CS, et al. Including walnuts in a low-fat/ modified-fat diet improves HDL cholesterol-to-total cholesterol ratios in patients with type 2 diabetes. Diabetes Care 2004;27:2777-83.

40 Mozaffarian D, Micha R, Wallace S. Effects on coronary heart disease of increasing polyunsaturated fat in place of saturated fat: a systematic review and meta-analysis of randomized controlled trials. PLoS Med 2010;7:e1000252.

41 Tapsell LC, Batterham MJ, Teuss G, et al. Long-term effects of increased dietary polyunsaturated fat from walnuts on metabolic parameters in type II diabetes. Eur J Clin Nutr 2009;63:1008-15.

42. Batterham M, Tapsell LC, Charlton KE. Predicting dropout in dietary weight loss trials using demographic and early weight change characteristics: implications for trial design. Obes Res Clin Pract 2016;10:189-96.

43. Wibisono C, Probst $Y$, Neale E, et al. Impact of food supplementation on weight loss in randomised-controlled dietary intervention trials: a systematic review and meta-analysis. Br J Nutr 2016;115:1406-14.
44. Weiss EP, Albert SG, Reeds DN, et al. Effects of matched weight loss from calorie restriction, exercise, or both on cardiovascular disease risk factors: a randomized intervention trial. Am J Clin Nutr 2016;104:576-86.

45. Bray GA. Weight management and exercise: any advantage? Am J Clin Nutr 2016;104.

46. Zazpe I, Sanchez-Tainta A, Estruch R, et al. A large randomized individual and group intervention conducted by registered dietitians increased adherence to Mediterranean-type diets: the PREDIMED study. J Am Diet Assoc 2008;108:1134-44.

47. Estruch R, Ros E, Salas-Salvadó J, et al. Primary prevention of cardiovascular disease with a mediterranean diet. $N$ Engl J Med 2013;368:1279-90.

48. Australian Bureau of Statistics. Census of Population and Housing, 2011, 2011.

49. Dawson JA, Kaiser KA, Affuso O, et al. Rigorous control conditions diminish treatment effects in weight loss-randomized controlled trials. Int J Obes 2016;40:895-8.

50. Baer DJ, Gebauer SK, Novotny JA. Walnuts consumed by healthy adults provide less available energy than predicted by the Atwater factors. J Nutr 2016;146.

51. Barr SB, Wright JC. Postprandial energy expenditure in whole-food and processed-food meals: implications for daily energy expenditure. Food Nutr Res 2010;54.

52. Flores-Mateo G, Rojas-Rueda D, Basora J, et al. Nut intake and adiposity: meta-analysis of clinical trials. Am J Clin Nutr 2013;97:1346-55.

53. Appel LJ, Sacks FM, Carey VJ, et al. Effects of protein, monounsaturated fat, and carbohydrate intake on blood pressure and serum lipids: results of the OmniHeart randomized trial. JAMA 2005;294:2455-64.

54. Batterham MJ, Tapsell LC, Charlton KE. Analyzing weight loss intervention studies with missing data: which methods should be used? Nutrition 2013;29(7-8):1024-9.

55. Moroshko I, Brennan L, O'Brien P. Predictors of dropout in weight loss interventions: a systematic review of the literature. Obes Rev 2011:12:912-34.

56. Nichols GA, Bell K, Kimes TM, et al. Medical Care costs associated with Long-term Weight Maintenance Versus Weight Gain among Patients with type 2 Diabetes. Diabetes Care 2016;39.

57. Australian Government Department of Health. Medicare Benefits Schedule Book, 2016.

58. Hyman DJ, Pavlik VN, Taylor WC, et al. Simultaneous vs sequential counseling for multiple behavior change. Arch Intern Med 2007:167:1152-8.

59. Niiranen TJ, Leino K, Puukka P, et al. Lack of impact of a comprehensive intervention on hypertension in the primary care setting. Am J Hypertens 2014;27:489-96.

60. Manore MM, Hand RK, Liguori G, et al. Knowledge and Beliefs That Promote or Hinder Collaboration among Registered Dietitian Nutritionists and Certified Exercise Professionals-Results of a Survey. J Acad Nutr Diet 2017;117:280-96. 